PROCEEDINGS OF THE

AMERICAN MATHEMATICAL SOCIETY

Volume 130, Number 6, Pages 1809-1817

S 0002-9939(01)06244-X

Article electronically published on October 12, 2001

\title{
FINITENESS THEOREMS FOR SUBMERSIONS AND SOULS
}

\author{
KRISTOPHER TAPP
}

(Communicated by Wolfgang Ziller)

\begin{abstract}
The first section of this paper provides an improvement upon known finiteness theorems for Riemannian submersions; that is, theorems which conclude that there are only finitely many isomorphism types of fiber bundles among Riemannian submersions whose total spaces and base spaces both satisfy certain geometric bounds. The second section of this paper provides a sharpening of some recent theorems which conclude that, for an open manifold of nonnegative curvature satisfying certain geometric bounds near its soul, there are only finitely many possibilities for the isomorphism class of a normal bundle of the soul. A common theme to both sections is a reliance on basic facts about Riemannian submersions whose $A$ and $T$ tensors are both bounded in norm.
\end{abstract}

\section{Counting Riemannian submersions}

In this section we improve upon known theorems which say that there are only finitely many equivalence classes of Riemannian submersions whose base space and total space both satisfy fixed geometric bounds. We consider two Riemannian submersions, $\pi_{1}: M_{1} \rightarrow B_{1}$ and $\pi_{2}: M_{2} \rightarrow B_{2}$, to be $C^{k}$-equivalent if there exists a $C^{k}$ diffeomorphism $f: M_{1} \rightarrow M_{2}$ which maps the fibers of $\pi_{1} C^{k}$-diffeomorphically onto the fibers of $\pi_{2}$. Every Riemannian submersion is a fiber bundle (as long as the total space is complete), and $C^{0}$-equivalence just means equivalence up to fiber bundle isomorphism.

The following theorem was proven by the author:

Theorem 1 ([10, Theorem 5.1]). Let $n, k \in \mathbb{Z}$ and $V, D, \lambda \in \mathbb{R}$. Then there are only finitely many $C^{1}$-equivalence classes in the set of Riemannian submersions $\pi: M^{n+k} \rightarrow B^{n}$ for which:

(1) $\operatorname{vol}(B) \geq V, \operatorname{diam}(B) \leq D,|\sec (B)| \leq \lambda$.

(2) $\operatorname{vol}(M) \geq V, \operatorname{diam}(M) \leq D,|\sec (M)| \leq \lambda$.

(3) $B$ is simply connected.

Here "vol", "diam", and "sec" are shorthand for volume, diameter, and sectional curvature. We improve this theorem by dropping condition (3) and also (at the cost of proving only $C^{0}$-finiteness) dropping the upper curvature bound on $M$ :

Received by the editors November 16, 2000 and, in revised form, December 15, 2000.

1991 Mathematics Subject Classification. Primary 53C20.

Key words and phrases. Soul, vector bundle, finiteness theorem, Riemannian submersion.

(C)2001 American Mathematical Society 
Theorem 2. Let $n, k \in \mathbb{Z}$ and $V, D, \lambda \in \mathbb{R}$. Assume $k \geq 4$. Then there are only finitely many $C^{0}$-equivalence classes in the set of Riemannian submersions $\pi: M^{n+k} \rightarrow B^{n}$ for which:

(1) $\operatorname{vol}(B) \geq V, \operatorname{diam}(B) \leq D,|\sec (B)| \leq \lambda$.

(2) $\operatorname{vol}(M) \geq V, \operatorname{diam}(M) \leq D, \sec (M) \geq-\lambda$.

Notice that some of the bounds in Theorems 1 and 2 are redundant. For example, since Riemannian submersions are curvature non-decreasing, the lower curvature bound on $B$ is redundant to the lower curvature bound on $M$. Also, the upper diameter bound on $B$ is redundant to the upper diameter bound on $M$.

Our proof of Theorem 2 will rely on the following finiteness theorem of $\mathrm{Wu}[12]$ :

Theorem 3 (J.Y. Wu). Let $B^{n}$ be a compact Riemannian manifold. Let $k \in \mathbb{Z}$ and $V, D, \lambda \in \mathbb{R}$. Assume $k \geq 4$. Then there are only finitely many $C^{0}$-equivalence classes in the set of Riemannian submersions $\pi: M^{n+k} \rightarrow B^{n}$ for which:

(1) $\sec (M) \geq \lambda$.

(2) For each fiber $F_{p}=\pi^{-1}(p), \operatorname{vol}\left(F_{p}\right) \geq V, \operatorname{diam}\left(F_{p}\right) \leq D, \sec \left(F_{p}\right) \geq \lambda$.

(3) Each fiber $F_{p}$ is totally convex.

Aside from Wu's theorem, the most important ingredient in our proof of Theorem 2 will be a result of the author from [10] which compares the intrinsic distance function, $d_{F_{p}}$, on a fiber $F_{p}=\pi^{-1}(p)$ of a Riemannian submersion $\pi: M \rightarrow B$ to the distance function $d_{M}$ of $M$ restricted to $F_{p}$ :

Lemma 4. (Folding of the fibers bound) Let $\pi: M^{n+k} \rightarrow B^{n}$ be a Riemannian submersion. Assume that the $A$ and $T$ tensor of $\pi$ are bounded in norm: $|A| \leq C_{A}$ and $|T| \leq C_{T}$.

(1) If $B$ is simply connected, then there exists $C$ depending only on $\left\{k, C_{A}, C_{T}, B\right\}$ such that $d_{F_{p}}(x, y) \leq C \cdot d_{M}(x, y)$ for all $p \in B$ and all $x, y \in F_{p}$.

(2) Even if $B$ is not simply connected, there exists $C$ depending on $\left\{k, C_{A}, C_{T}, B\right\}$ such that $d_{F_{p}}(x, y) \leq C \cdot d_{M}(x, y)$ for all $p \in B$ and all $x, y \in F_{p}$ with $d_{M}(x, y)<\operatorname{inj}(B)$.

Here $\operatorname{inj}(B)$ denotes the injectivity radius of $B$. Part (1) of Lemma 4 was proven in [10, Thm. 5.1], and part (2) is immediate from the proof of part (1), since the simple connectivity of $B$ is only used to insure that the image under $\pi$ of a minimal path in $M$ between $x$ and $y$ is nulhomotopic in $B$.

As mentioned in [10], one can use O'Neill's formula and an argument of Perelman to bound the $A$ and $T$ tensor of a submersion in terms of geometric bounds for $M$ and $B$ :

Lemma 5. Let $\pi: M \rightarrow B$ be a Riemannian submersion.

(1) The $A$ tensor is bounded in norm by a constant $C_{A}$ depending only on a lower curvature bound for $M$ and an upper curvature bound for $B$ (if such bounds exist).

(2) The $T$ tensor is bounded in norm by a constant $C_{T}$ depending only on a lower curvature bound for $M$ and a lower injectivity radius bound for $B$ (if such bounds exist).

We use Lemmas 4 and 5 to generalize Wu's theorem:

Proposition 6. Theorem 3 remains true if hypothesis (3) is removed. 
Proof. Hypothesis (3) says that $d_{F_{p}}=d_{M}$ for all $p \in B$. It follows immediately from Wu's proof that hypothesis (3) can be replaced by the following weaker assumption:

$\left(3^{\prime}\right)$ For any $p \in B$, if $x, y \in F_{p}$ and $d_{M}(x, y)<K$, then $d_{F_{p}}(x, y) \leq C \cdot d_{M}(x, y)$, where $C$ and $K$ are any fixed constants. But assumption $\left(3^{\prime}\right)$ is automatic by Lemmas 4 and 5

Wu states in [12, p. 513] that his theorem remains true if "Riemannian submersion" is replaced by " $L$-Lipschitz map", where $L$ is any fixed number. It is not clear to us that his proof goes through in this generality; however, it is obvious that the proof still holds when "Riemannian submersion" is replaced by "submersion $\pi$ for which $\left.\pi_{*}\right|_{\mathcal{H}}$ is at every point an $L$-biLipschitz linear map", where $\mathcal{H}$ denotes the distribution orthogonal to the fibers. We will call such a submersion an " $L$ Riemannian submersion". In review, the following generalization of Wu's Theorem is true:

Proposition 7 (Generalization of Wu's Theorem). Suppose $B^{n}$ is a compact Riemannian manifold. Let $k \in \mathbb{Z}$ and $V, D, \lambda, L \in \mathbb{R}$. Assume $k \geq 4$. Then there are only finitely many $C^{0}$-equivalence classes in the set of $L$-Riemannian submersions $\pi: M^{n+k} \rightarrow B^{n}$ for which:

(1) $\sec (M) \geq \lambda$.

(2) For each fiber $F_{p}, \operatorname{vol}\left(F_{p}\right) \geq V, \operatorname{diam}\left(F_{p}\right) \leq D, \sec \left(F_{p}\right) \geq \lambda$.

We now prove Theorem 2, essentially by proving that a Riemannian submersion satisfying its hypotheses will automatically satisfy the hypotheses of the above generalization of Wu's theorem.

Proof of Theorem Q Let $\mathfrak{M}$ denote the set of all candidates for the base space (that is, all Riemannian $n$-manifolds satisfying vol $\geq V$, diam $\leq D$, and $|\sec | \leq \lambda$ ). Choose $\left\{B_{0}, \ldots, B_{l}\right\} \subset \mathfrak{M}$ and $L \in \mathbb{R}$ such that for each $B \in \mathfrak{M}$, there exists an $L$-biLipschitz diffeomorphism $f: B \rightarrow B_{i}$ for some $i$. This is possible because $\mathfrak{M}$ is precompact in the Lipschitz topology and contains only finitely many diffeomorphism types; see for example [9].

Now if $\pi: M \rightarrow B$ is a Riemannian submersion satisfying the hypotheses of Theorem 2, then $\bar{\pi}=f \circ \pi: M \rightarrow B_{i}$ is an $L$-Riemannian submersion. Further, the two submersions, $\pi$ and $\bar{\pi}$, are isomorphic as fiber bundles. Let $p^{\prime} \in B_{i}$ and let $p=f^{-1}\left(p^{\prime}\right) \in B$. We will argue that the fiber $F_{p}=\pi^{-1}(p)=\bar{\pi}^{-1}\left(p^{\prime}\right)$ satisfies a curvature, diameter, and volume bound depending only on $\{V, D, \lambda, k, n\}$. Then, applying Theorem 7 once for each of the base spaces $B_{0}, \ldots, B_{l}$ finishes the proof.

First, by Lemma [5 the $A$ and $T$ tensors of $\pi$ are bounded in norm: $|A| \leq C_{A}$ and $|T| \leq C_{T}$. A well-known result of Cheeger provides a lower injectivity radius bound for manifolds in $\mathfrak{M}$, which means that $C_{A}$ and $C_{T}$ depend only on $\{V, D, \lambda, n\}$. By the Gauss equations, the fibers of $\pi$ satisfy a lower curvature bound depending on $C_{T}$ and $\lambda$.

Second, to control $\operatorname{vol}\left(F_{p}\right)$, notice that any two fibers have similar volumes. More precisely, the diffeomorphism $h^{\alpha}: F_{p_{1}} \rightarrow F_{p_{2}}$ associated to a minimal path, $\alpha$, in $B$ between $p_{1}$ and $p_{2}$ satisfies the Lipschitz constant $e^{C_{T} \cdot \operatorname{length}(\alpha)} \leq e^{C_{T} \cdot D}$ (see [6] Lemma 4.2]), so $\operatorname{vol}\left(F_{p_{1}}\right) \leq\left(e^{k C_{T} \cdot D}\right) \cdot \operatorname{vol}\left(F_{p_{2}}\right)$. But by Fubini's theorem, $\operatorname{vol}(M)=$ $\int_{q \in B} \operatorname{vol}\left(F_{q}\right) \operatorname{dvol}_{B}$, which implies that for any $q \in B, \operatorname{vol}\left(F_{q}\right) \geq \frac{\operatorname{vol}(M)}{\operatorname{vol}(B)}\left(e^{-k C_{T} \cdot D}\right)$. By the Bishop-Gromov inequality, $\operatorname{vol}(B)$ is bounded above by a constant depending 
only on $D, \lambda$, and $n$. This observation completes our argument that $\operatorname{vol}\left(F_{p}\right)$ is bounded below.

Third, we establish an upper bound for $\operatorname{diam}\left(F_{p}\right)$. By part (2) of Lemma 4 there exists $C$ depending only on $\left\{k, C_{A}, C_{T}, B\right\}$ such that $d_{F_{p}}(x, y) \leq C \cdot d_{M}(x, y)$ for all $p \in B$ and all $x, y \in F_{p}$ with $d_{M}(x, y)<\operatorname{inj}(B)$. In fact, it is argued in 10, page 10] that $C$ can be chosen to depend only on $\left\{k, C_{A}, C_{T}\right\}$ and the assumed geometric bounds for $B$. Now suppose $\gamma:[0, l] \rightarrow F_{p}$ is a minimizing unit-speed geodesic in $F_{p}$. We wish to bound its length $l$. The points $p_{i}=\gamma\left(\frac{i}{2} \operatorname{inj}(B)\right)$ are equally spaced along $\gamma$. Let $B_{i}$ be the ball in $M$ centered at $p_{i}$ with radius $r=\frac{1}{4 C} \operatorname{inj}(B)$. The above bound on the folding of the fibers implies that the balls $B_{i}$ are disjoint (if $B_{i} \cap B_{j} \neq \emptyset$, then $d_{M}\left(p_{i}, p_{j}\right)<\frac{1}{2 C} \operatorname{inj}(B)$, so $\left.d_{F_{p}}\left(p_{i}, p_{j}\right)<\frac{1}{2}(\operatorname{inj}(B))\right)$. But the geometric bounds on $M$ imply a bound on the number of disjoint balls of any fixed radius which can be packed into $M$. This provides a bound for $l$, and proves the theorem.

We do not know if Theorem 2 is true without the upper curvature bound on $B$.

\section{Counting NORMAl BUndLes OF SOUls}

In this section we review and improve upon some recent theorems which state that, for an open manifold of nonnegative curvature satisfying certain geometric bounds near its soul, there are only finitely many possibilities for the isomorphism class of the normal bundle of the soul. The first theorem in this direction is due to Guijarro and Walschap [5]:

Theorem 8 (Guijarro-Walschap). Fix $n, k \in \mathbb{Z}$ and $\Lambda, R \in \mathbb{R}$. Suppose $M^{n+k}$ is an open manifold of nonnegative curvature with soul, $\Sigma$, isometric to the $n$ dimensional unit round sphere. If for some point $p \in \Sigma$, the ball about $p$ in $M$ of radius $R$ has sectional curvatures $\leq \Lambda$, then there are only finitely many possibilities for the isomorphism class of the normal bundle of the soul.

It turns out that the most interesting aspect of this theorem is the fact the upper curvature bound is only required near a single point of the soul, and not near every point of the soul. In fact, the following theorem is much simpler to prove:

Proposition 9. Fix $k \in \mathbb{Z}$ and $\Lambda \in \mathbb{R}$. Let $\Sigma^{n}$ be a compact Riemannian manifold with nonnegative curvature. Suppose $M^{n+k}$ is an open manifold of nonnegative curvature with soul isometric to $\Sigma$. If the vertical curvatures at every point of the soul are $\leq \Lambda$, then there are only finitely many possibilities for the isomorphism class of the normal bundle of the soul.

Here "vertical curvatures" means the curvatures of planes spanned by 2 vectors which are both normal to the soul.

Proof. The bound on the vertical curvatures at points of $\Sigma \subset M$ implies a bound on the norm of the curvature tensor $R^{\nabla}$ associated to the connection $\nabla$ in the normal bundle $\nu(\Sigma)$ of $\Sigma$; this follows from [11] page 615]. Chern-Weil theory therefore provides bounds on all characteristic classes of the bundle. But the characteristic classes classify the bundle up to finite ambiguity; see for example [1, Chapter 7]. The result follows. 
Example 10. There is a countable collection of rank 2 vector bundles over $S^{2}$, which can be described as $M_{k}:=\left(S^{3} \times \mathbb{R}^{2}\right) / S^{1}, k \in \mathbb{Z}^{+}$, where $S^{1}$ acts diagonally as

$$
(p, v) \stackrel{\theta}{\mapsto}\left(e^{i \theta} \cdot p, e^{-k i \theta} \cdot v\right) .
$$

A calculation shows that at any point $p$ of the soul of $M_{k},\left|R^{\nabla}\right|=2 k$, and the vertical curvatures at the souls of $M_{k}$ also grow linearly in $k$.

Instead of fixing $\Sigma$ in Proposition 9, one need only fix geometric bounds on $\Sigma$ :

Corollary 11. Fix $n, k \in \mathbb{Z}$ and $\Lambda, V, D, \lambda \in \mathbb{R}$. Suppose $M^{n+k}$ is an open manifold of nonnegative curvature with soul $\Sigma^{n}$ such that:

(1) $\operatorname{vol}(\Sigma) \geq V, \operatorname{diam}(\Sigma) \leq D, \sec (\Sigma) \leq \lambda$.

(2) The vertical curvatures at every point of $\Sigma$ are $\leq \Lambda$.

Then there are only finitely many possibilities for the isomorphism class of the normal bundle of the soul.

Proof. As in our proof of Theorem[2] let $\mathfrak{M}$ denote the set of all candidates for $\Sigma$ (that is, all Riemannian $n$-manifolds satisfying vol $\geq V$, diam $\leq D$, and $0 \leq \sec \leq$ $\lambda)$. Choose $\left\{B_{0}, \ldots, B_{l}\right\} \subset \mathfrak{M}$ and $L \in \mathbb{R}$ such that for each $B \in \mathfrak{M}$, there exists an $L$-biLipschitz diffeomorphism $f: B_{i} \rightarrow B$ for some $i$. Any vector bundle over any manifold $B \in \mathfrak{M}$ which admits a connection satisfying the bound $\left|R^{\nabla}\right| \leq C$ is isomorphic to a vector bundle over some $B_{i}$ which admits a connection satisfying $\left|R^{\nabla}\right| \leq L^{2} \cdot C$; namely, the pull-back (via $f$ ) of the bundle with the pull-back connection. So applying the argument of Proposition 9 once for each base space $B_{i}$ completes the proof.

Belegradek and Kapovitch [2] recently obtained the following:

Theorem 12 (Belegradek-Kapovitch). Let $n, k \in \mathbb{Z}$ and $D, \Lambda, R \in \mathbb{R}$. Suppose that $M^{n+k}$ is an open manifold of nonnegative curvature with soul $\Sigma^{n}$ for which:

(1) $\operatorname{diam}(\Sigma) \leq D$.

(2) For some $p \in \Sigma$, $\operatorname{vol}_{M}(B(p, R)) \geq \Lambda$.

Then there are only finitely many possibilities for the isomorphism class of the normal bundle of the soul.

Here $B(p, R)$ denotes the metric ball about $p$ in $M$ with radius $R$. In the remainder of this section we prove the following generalization of Theorem 8 and Proposition 9:

Theorem 13. Fix $k \in \mathbb{Z}$ and $\Lambda \in \mathbb{R}$. Let $\Sigma^{n}$ be a compact Riemannian manifold with nonnegative curvature. Suppose $M^{n+k}$ is an open manifold of nonnegative curvature with soul isometric to $\Sigma^{n}$. Assume either one (not both) of the following hypotheses:

(1) The vertical curvatures at every point of the soul are $\leq \Lambda$.

(2) There exists a point $p$ of the soul such that the curvatures of all radial planes inside $B(p, R)$ are $\leq \Lambda$, where $R=\pi / 2 \sqrt{\Lambda}$.

Then the number of possibilities for the isomorphism class of the normal bundle of the soul is $\leq C \cdot(1+\Lambda)^{c}$, where $c$ and $C$ depend on $\left\{\Sigma^{n}, k\right\}$. 
A "radial plane" means a plane tangent to the Sharafutdinov fibers which contains the gradient of the distance to the soul. In version 1 of the theorem (the version with the first hypothesis), the fact that there are only finitely many possible bundles was shown in Proposition 9, but we do not believe that our polynomial bound is obtainable using the argument by which Proposition 9 was proven. In version 2 of the theorem, since only radial curvatures are assumed to be bounded, the fact that there are only finitely many possible bundles does not follow from Belegradek and Kapovitch's result. Our proof is modeled after Guijarro and Walschap's proof of Theorem 8 , and uses the following result of Gromov [4, 0.5.E]:

Lemma 14 (Gromov). Let $X$ and $Y$ be compact Riemannian manifolds. Assume that $Y$ has finite fundamental group. Then the number of homotopy classes of maps $X \rightarrow Y$ which admit an L-Lipschitz representative is $\leq A \cdot(1+L)^{a}$, where the exponent a depends only on the homotopy types of $X$ and $Y$, while the constant $A$ depends on their metrics as well.

Proof of version 1 of Theorem 13. Let $M$ be an open manifold with nonnegative sectional curvature and soul $\Sigma \subset M$. Assume that all vertical curvatures at all points of $\Sigma$ are $\leq \Lambda$. This implies that the curvature tensor, $R^{\nabla}$, of the connection $\nabla$ in the normal bundle $\nu(\Sigma)$ of $\Sigma$ is bounded in norm as $\left|R^{\nabla}\right| \leq \bar{\Lambda}$, where $\bar{\Lambda}$ depends on $\Lambda$ and on an upper section curvature bound, $\lambda$, for $\Sigma$; see [11, page 615]. In fact, $\bar{\Lambda}$ is linear in $\Lambda$.

Let $E$ denote the total space of the normal bundle $\nu(\Sigma)$, and let $\pi: E \rightarrow \Sigma$ denote the projection. We begin by choosing an explicit atlas of local trivializations for $\nu(\Sigma)$. Choose a value $r<\frac{1}{2} \min \left\{\operatorname{inj}(\Sigma), \frac{\pi}{\sqrt{\lambda}}\right\}$. Choose a finite collection $\left\{p_{1}, \ldots, p_{l}\right\}$ of points of $\Sigma$ such that the collection of $r$-balls $\left\{B_{i}:=B\left(p_{i}, r\right)\right\}$ covers $\Sigma$. Let $\left\{\eta_{i}\right\}$ denote a partition of unity subordinate to $\left\{B_{i}\right\}$.

The connection, $\nabla$, provides a natural collection of local trivializations, $\Phi_{i}: B_{i} \times$ $\mathbb{R}^{k} \rightarrow \pi^{-1}\left(B_{i}\right)$. More precisely, for each $i$, choose a fixed orthogonal identification $s_{i}: \mathbb{R}^{k} \rightarrow E_{p_{i}}:=\pi^{-1}\left(p_{i}\right)$, and define $\Phi_{i}(q, v)$ to be the parallel transport of $s_{i}(v)$ along the minimal geodesic, $\gamma_{q}^{i}$, between $p_{i}$ and $q$. That is, $\Phi_{i}(q, v):=P_{\gamma_{q}^{i}}\left(s_{i}(v)\right)$. Also define $h_{i}: \pi^{-1}\left(B_{i}\right) \rightarrow \mathbb{R}^{k}$ as $h_{i}:=\Pi_{i} \circ \Phi_{i}^{-1}$, where $\Pi_{i}: B_{i} \times \mathbb{R}^{k} \rightarrow \mathbb{R}^{k}$ denotes the projection onto the second factor. Notice that $h_{i}(v)=s_{i}^{-1}\left(P_{\left(\gamma_{\pi(v)}^{i}\right)^{-1}}(v)\right)$.

For any pair $(i, j)$ for which $B_{i j}:=B_{i} \cap B_{j}$ is nonempty, we have a transition function $g_{i j}: B_{i j} \rightarrow O(k)$, defined as

$$
g_{i j}(q)(v):=h_{j}\left(h_{i}^{-1}(v) \cap \pi^{-1}(q)\right)=\left(s_{j}^{-1} \circ P_{\left(\gamma_{q}^{j}\right)^{-1}} \circ P_{\gamma_{q}^{i}} \circ s_{i}\right)(v) .
$$

We wish to establish a Lipschitz bound on each $g_{i j}$. Suppose that $q \in B_{i j}$, and let $X \in T_{q} \Sigma$ with $|X|=1$. Let $q(s):=\exp (s X)$ and let $v \in \mathbb{R}^{k}$ be any unit-length vector. Let $\tau_{v}(s):=g_{i j}(q(s))(v)$, which is a path on the unit sphere of $\mathbb{R}^{k}$. In order to bound $\left|\left(g_{i j}\right)_{*}(X)\right|$, it will clearly suffice to find a bound on $\left|\tau_{v}^{\prime}(0)\right|$ which does not depend on the choice of $v$.

For small values of $s$, let $t \rightarrow \sigma_{s}(t)=\sigma(s, t)$ be a minimal unit-speed path between $p_{i}$ and $q(s)$ followed by a minimal unit-speed path between $q(s)$ and $p_{j}$. By construction, $\tau_{v}(s)=\left(s_{j}^{-1} \circ P_{\sigma_{s}} \circ s_{i}\right)(v)$. Let area $\left(s_{0}\right)$ denote the area of the homotopy between $\sigma_{0}$ and $\sigma_{s_{0}}$ determined by the family of curves $\sigma_{s}, s \in\left[0, s_{0}\right]$. By [3] Proposition 6.2.1], $\angle\left(\tau_{v}(0), \tau_{v}\left(s_{0}\right)\right) \leq \bar{\Lambda} \cdot \operatorname{area}\left(s_{0}\right)$. Therefore $\left|\tau_{v}^{\prime}(0)\right| \leq \bar{\Lambda}$. $\operatorname{area}^{\prime}(0)$. But area $(0)=\int_{t=0}^{\operatorname{dist}\left(p_{i}, p_{j}\right)}\left|\frac{\partial}{\partial s} \sigma(0, t)\right| \mathrm{dt} \leq \operatorname{dist}\left(p_{i}, p_{j}\right) \leq 2 r$. To see the first inequality, notice that the vector field $\frac{\partial}{\partial s} \sigma$ is a Jacobi field along $\gamma_{q}^{i}$ and along 
$\gamma_{q}^{j}$, and that both Jacobi fields have norms which vary monotonically between 0 and $|X|=1$. Therefore, $\left|\tau_{v}^{\prime}(0)\right| \leq 2 r \bar{\Lambda}$, which implies that $\left|\left(g_{i j}\right)_{*}(X)\right| \leq 2 k r \bar{\Lambda}$. In other words, each transition function $g_{i j}$ is $(2 k r \bar{\Lambda})$-Lipschitz.

The isomorphism class of the vector bundle $E \stackrel{\pi}{\rightarrow} \Sigma$ is determined by the homotopy type of its classifying map $f: \Sigma \rightarrow G_{k}\left(\mathbb{R}^{N}\right)$, where $G_{k}\left(\mathbb{R}^{N}\right)$ denotes the Grassmannian of $k$-dimensional subspaces of $\mathbb{R}^{N}$, and $N=k \cdot l$. The map $f$ can be described explicitly in terms of our chosen atlas of trivializations of the bundle as follows (see for example [7, Theorem 3.5.5]): think of $\mathbb{R}^{N}=\sum_{1}^{l} \mathbb{R}^{k}$, and define

$$
f(p):=\left\{\left(\eta_{1}(p) \cdot h_{1}(v), \ldots, \eta_{l}(p) \cdot h_{l}(v)\right) \mid v \in \pi^{-1}(p)\right\} .
$$

Our goal is to establish a Lipschitz bound for $f$ which is linear in $\Lambda$. For this purpose, the most useful description of the homogeneous metric on $G_{k}\left(\mathbb{R}^{N}\right)$ is as follows. Fix orthonormal bases of $\mathbb{R}^{N}$ and $\mathbb{R}^{k}$. Let $\mathrm{I}_{k}$ denote the $k \times k$ identity matrix, and let $\mathrm{I}_{k}^{N}:=\left(\begin{array}{c}\mathrm{I}_{k} \\ 0\end{array}\right)$ denote the $N \times k$ matrix whose top $k$ rows form $\mathrm{I}_{k}$, and whose bottom $N-k$ rows are zeros. An arbitrary point, $V$, of $G_{k}\left(\mathbb{R}^{N}\right)$ can be described as $V=\operatorname{im}\left(P \cdot \mathrm{I}_{k}^{N}\right)$ for some $P \in O(N)$. An arbitrary path, $\gamma(t)$, with $\gamma(0)=V$ can be described as $\gamma(t)=\operatorname{im}(P \cdot A(t))$, where $A(t): \mathbb{R}^{k} \rightarrow \mathbb{R}^{N}$ is a family of linear embeddings with $A(0)=\mathrm{I}_{k}^{N}$. Then $\left|\gamma^{\prime}(0)\right|^{2}=\operatorname{trace}\left(B^{T} \cdot B\right)$, where $B:=A^{\prime}(0)-\mathrm{I}_{k}^{N} \cdot\left(\mathrm{I}_{k}^{N}\right)^{T} \cdot A^{\prime}(0)$ (that is, $B$ is obtained from the matrix $A^{\prime}(0)$ by replacing the top $k$ rows with zeros). In particular, if all components $a_{i j}(t)$ of $A(t)$ satisfy $\left|a_{i j}^{\prime}(0)\right| \leq c$, then $\left|\gamma^{\prime}(0)\right| \leq k c$.

Now fix $q \in B_{1}$ and $X \in T_{q} \Sigma$ with $|X|=1$, and let $q(t)=\exp (t X)$. Let $A(t)=\left(a_{i j}(t)\right): \mathbb{R}^{k} \rightarrow \mathbb{R}^{N}$ be defined as

$$
A(t)(v):=\left(\eta_{1}(q(t)) \cdot v, \eta_{2}(q(t)) \cdot g_{12}(q(t))(v), \ldots, \eta_{l}(q(t)) \cdot g_{1 l}(q(t))(v)\right) .
$$

It is clear that $f(q(t))=\operatorname{im}(A(t))$. Choose $S=\left(s_{i j}\right): \mathbb{R}^{k} \rightarrow \mathbb{R}^{k}$ such that the matrix $A(0) \cdot S$ is an orthogonal map onto its image. That way, $P \cdot A(0) \cdot S=\mathrm{I}_{k}^{N}$ for some $P=\left(p_{i j}\right) \in O(N)$. Since $A(0)$ already maps an orthonormal basis of $\mathbb{R}^{k}$ onto an orthogonal set in $\mathbb{R}^{N}, S$ can be chosen as a diagonal matrix, and it is clear that the components of $S$ satisfy $\left|s_{i j}\right| \leq k . f(q(t))=\operatorname{im}(A(t))=\operatorname{im}\left(P^{-1} P \cdot A(t) \cdot S\right)$. So, by the previous discussion of the homogeneous metric on $G_{k}\left(\mathbb{R}^{N}\right)$

$$
\begin{aligned}
\left|f_{*}(X)\right| \leq & k \cdot \max \left|\left((P \cdot A \cdot S)^{\prime}(0)\right)_{i j}\right| \\
\leq & k^{2} N\left(\max \left|p_{i j}\right|\right) \cdot\left(\max \left|a_{i j}^{\prime}(0)\right|\right) \cdot\left(\max \left|s_{i j}\right|\right) \\
= & k^{3} N\left(\max \left|a_{i j}^{\prime}(0)\right|\right) \\
\leq & k^{3} N\left[\left(\max \left|\left(\eta_{i}\right)_{*}(X)\right|\right) \cdot\left(\max \left|\left(g_{1 s}\right)_{i j}(q)\right|\right)\right. \\
& +\left(\max \mid\left(\eta_{i}(q) \mid\right) \cdot\left(\max \left|\left(\left(g_{1 s}\right)_{i j}\right)_{*}(X)\right|\right)\right] \\
\leq & k^{3} N(\hat{L} \cdot 1+1 \cdot 2 r \bar{\Lambda})=k^{3} N(\hat{L}+2 r \bar{\Lambda}),
\end{aligned}
$$

where $\hat{L}$ denotes a Lipschitz bound on the functions $\eta_{i}$.

Thus, $f$ satisfies the Lipschitz constant $L:=k^{3} N(\hat{L}+2 r \bar{\Lambda})$. The homotopy type of this map classifies the bundle, so the proof is completed by Lemma 14

Next we prove version 2 of Theorem 13 which relies on the following result found in [10]:

Lemma 15 (10, Lemma 3.3]). Let $\pi: M^{n+k} \rightarrow B^{n}$ be a Riemannian submersion whose $A$ and $T$ tensors are both bounded in norm; $|A| \leq C_{A}$ and $|T| \leq C_{T}$. Let $\alpha_{s}(t)=\alpha(s, t)(s \in[0, \epsilon), t \in[0,1])$ denote a family of piecewise-smooth paths in 
$B$ with fixed endpoints: $\alpha_{s}(0)=p_{1}, \alpha_{s}(1)=p_{2}$. Assume $\left|\alpha_{0}^{\prime}(t)\right| \leq C_{1}$. Assume for the variational vector field $V(t):=\frac{\partial}{\partial s} \alpha(0, t)$ along $\alpha_{0}$ that $|V(t)| \leq C_{2}$. Let $x \in F_{p_{1}}=\pi^{-1}\left(p_{1}\right)$. For each fixed $s$, let $t \mapsto \bar{\alpha}_{s}(t)=\bar{\alpha}(s, t)$ denote the horizontal lift the path $t \mapsto \alpha_{s}(t)$ with $\bar{\alpha}_{s}(0)=x$. Then $\varrho(s):=\bar{\alpha}_{s}(1)$ is a path in the fiber $F_{p_{2}}$, and $\left|\varrho^{\prime}(0)\right| \leq C$, where $C$ depends on $C_{A}, C_{T}, C_{1}, C_{2}$ and $k$.

Proof of version 2 of Theorem 13. Let $M$ be an open manifold with nonnegative sectional curvature and soul $\Sigma \subset M$. Assume that at some point $p \in \Sigma$, the ball in $M$ at $p$ with radius $R=\pi / 2 \sqrt{\Lambda}$ has vertical curvatures $\leq \Lambda$. Let sh $: M \rightarrow \Sigma$ denote the Sharafutdinov map (see [8]), so that $\pi=s h \circ \exp$, where $\pi: E \rightarrow \Sigma$ denotes the normal bundle of $\Sigma$ as before, and exp $: E \rightarrow M$ denotes the normal exponential map. By Lemma 5 the $A$ and $T$ tensors of sh are bounded by constant depending only on $\Sigma$.

As in the previous proof, choose points $\left\{p_{i}\right\} \subset \Sigma$ and balls $\left\{B_{i}\right\}$ about these points. Define trivializations of $\nu(\Sigma), \Phi_{i}: B_{i} \times \mathbb{R}^{k} \rightarrow \pi^{-1}\left(p_{i}\right)$, and transition functions $g_{i j}: B_{i} \cap B_{j} \rightarrow O(k)$ exactly as before. Also as before, Choose $q \in B_{i j}$, $X \in T_{q} \Sigma$ with $|X|=1, v \in \mathbb{R}^{k}$ with $|v|=1$, let $q(s):=\exp (s X)$ and $\tau_{v}(s):=$ $g_{i j}(q(s))(v)$. Define the family of paths $\sigma_{s}(t)=\sigma(s, t)$ as before, so that $\tau_{v}(s)=$ $\left(s_{j}^{-1} \circ P_{\sigma_{s}} \circ s_{i}\right)(v)$. It is convenient to reparameterize so that $\alpha:[0, \epsilon] \times[0,1] \rightarrow \Sigma$.

We require a new argument to bound $\left|\tau_{v}^{\prime}(0)\right|$. For each fixed $s$, let $t \mapsto \bar{\alpha}_{s}(t)=$ $\bar{\alpha}(s, t)$ denote the horizontal lift the path $t \mapsto \alpha_{s}(t)$ with $\bar{\alpha}_{s}(0)=\exp \left(R \cdot s_{i}(v)\right)$. Then $\varrho(s)=\bar{\alpha}_{s}(1)$ is a path in the fiber $\operatorname{sh}^{-1}\left(p_{j}\right)$. By Lemma 15] $\left|\varrho^{\prime}(0)\right| \leq C$, where $C$ depends on $\Sigma, k$ and length $\left(\alpha_{0}\right)=\operatorname{dist}\left(p_{i}, p_{j}\right)$.

Notice that $v(s)=s_{j}\left(\tau_{v}(s)\right) \in \nu_{p_{j}}(\Sigma)$ defines a family of unit-length vectors normal to $\Sigma$ at $p_{j}$. For each $s$, let $a_{s}$ be the geodesic in the direction $v(s)$; that is, $a_{s}(t)=\exp (t \cdot v(s))$. Let $J(t)=\frac{\partial}{\partial s} a_{s}(t)$ be the corresponding Jacobi field along $a_{0}$. Notice that $a_{s}(R)=\varrho(s)$, which means that $|J(R)|=\left|\varrho^{\prime}(0)\right| \leq C$.

If $p_{j}$ happens to be the point $p$ about which we have an upper curvature bound $\Lambda$, then the Rauch Theorem says that $|\tilde{J}(R)| \leq|J(R)| \leq C$, where $\tilde{J}$ is a Jacobi field on the sphere of constant curvature $\Lambda$ with $\tilde{J}(0)=0$ and $\left|\tilde{J}^{\prime}(0)\right|=\left|J^{\prime}(0)\right|$. But,

$$
|\tilde{J}(R)|=\frac{\sin (R \sqrt{\Lambda})}{\sqrt{\Lambda}} \cdot\left|\tilde{J}^{\prime}(0)\right|=\frac{1}{\sqrt{\Lambda}} \cdot\left|\tilde{J}^{\prime}(0)\right| \leq C
$$

Therefore,

$$
\left|\tau_{v}^{\prime}(0)\right|=\left|J^{\prime}(0)\right|=\left|\tilde{J}^{\prime}(0)\right| \leq C \cdot \sqrt{\Lambda}
$$

When $\Lambda \geq 1,\left|\tau_{v}^{\prime}(0)\right| \leq C \cdot \Lambda$, which together with the argument of the previous proof proves the theorem.

On the other hand, if $p_{j}$ is different from $p$, then for each $s$, join a fixed minimal path in $\Sigma$ from $p_{j}$ to $p$ to the end of the path $\alpha_{s}$. Applying the argument above to this lengthened family of paths completes the proof.

\section{ACKNowledGement}

The author wishes to thank Wolfgang Ziller and Vitali Kapovitch for many helpful conversations about the results in this paper. 


\section{REFERENCES}

1. I. Belegradek, Counting nonnegatively curved manifolds, Ph.D. Thesis, University of Maryland, 1998.

2. I. Belegradek and V. Kapovitch, Finiteness theorems for maps into manifolds of bounded geometry, preprint.

3. P. Buser and H. Karcher, Gromov's almost flat manifolds, Société Mathématique de France, Astérisque 81, 1981. MR 83m:53070

4. M. Gromov, Carnot-Carathéodory spaces seen from within, Progress in Mathematics, Vol. 144, 1996. MR 2000f:53034

5. L. Guijarro and G. Walschap, Twisting and nonnegative curvature metrics on vector bundles over the sphere, J. Differential Geom. 52(1999), no. 1, 189-202. MR 2000m:53042

6. L. Guijarro and G. Walschap, The metric projection onto the soul, Tans. Amer. Math. Soc. 352 (2000), no. 1, 55-69. MR 2000c:53034

7. D. Husemoller, Fibre bundles, McGraw-Hill, 1966. MR 37:4821

8. G. Perelman, Proof of the soul conjecture of Cheeger and Gromoll, J. Differential Geom. 40 (1994), 209-212. MR 95d:53037

9. S. Peters, Convergence of Riemannian manifolds, Compositio Mathematica. 62 (1987)3-16. MR 88i:53076

10. K. Tapp, Bounded Riemannian submersions, Indiana Univ. Math. J. 49 (2000). CMP 2001:04

11. G. Walschap, Soul-preserving submersions, Michigan Math. J. 41 (1994), 609-617. MR 95h:53042

12. J.Y. Wu, A parameterized geometric finiteness theorem, Indiana Univ. Math. J. 45, no. 2 (1996), 511-528. MR 97h:53046

Department of Mathematics, State University of New York at Stony Brook, Stony BROOK, NEW YorK 11794-3651

E-mail address: ktapp@math.sunysb.edu 